\title{
Enlarging the basin of attraction by a uniting output feedback controller
}

\author{
Miguel A. Davó ${ }^{\mathrm{a}}$, Christophe Prieur ${ }^{\mathrm{a}}$, Mirko Fiacchini ${ }^{\mathrm{a}}$, Dragan Nešić ${ }^{\mathrm{b}}$, \\ ${ }^{\mathrm{a}}$ Univ. Grenoble Alpes, CNRS, GIPSA-lab, F-38000 Grenoble, France. \\ ${ }^{\mathrm{b}}$ Department of Electrical and Electronic Engineering, The University of Melbourne, Australia
}

\begin{abstract}
We consider a system for which two predesigned stabilizing controllers with bounded domains of attraction are known. One renders the system asymptotically stable with some desired performance, and the other provides ultimate boundedness with larger domain of attraction. Assuming that two subsets of the domains of attraction are known, one larger than the other, this work states the problem of combining both controllers with the goal of guaranteeing asymptotic stability properties in the largest subset while the desired performance is locally achieved. We design a switching logic between the controllers that solves the problem, based on the existence of a local tunable observer. The resulting control law is defined by a hybrid output feedback controller. The effectiveness of the proposed solution is illustrated by a numerical example.
\end{abstract}

Key words: hybrid system, stability analysis

\section{Introduction}

There is a multitude of techniques to design asymptotically stabilizing control feedbacks laws. Nonetheless, most of the well known techniques (backstepping, feedback linearization, passivation, ...) usually do not address the problem of system performance. It is of great importance to design control laws providing both asymptotic stability and guaranteed performance requirements. A simple local solution to this problem can be obtained, for instance, via linearization and then applying tools for linear systems. This leads to the idea of uniting two predesigned local and global controllers as proposed in [18], so that local performance objectives are achieved while global asymptotic stability is guaranteed.

Different strategies have been proposed to tackle the problem of uniting local and global controllers. A first algorithm for patching two controllers was presented

\footnotetext{
* This work has been supported by the LabEx PERSYVALLab (ANR-11-LABX-0025-01), the ANR project LimICoS contract number 12-BS03-005-01, and the Australian Research Council under the discovery grants scheme. Corresponding author M.A. Davó.

Email addresses: Miguel.Davo-Navarro@gipsa-lab.fr (Miguel A. Davó), mirko.fiacchini@gipsa-lab.fr (Christophe Prieur), Christophe.Prieur@gipsa-lab.fr (Mirko Fiacchini), dnesic@unimelb.edu.au (Dragan Nešić).
}

in [18] (see also $[11,12]$ ), and later applied to real experiments in [17]. The solution is given in the form of a continuous static time-invariant controller. However, considering general control systems, the uniting problem cannot be solved by only continuous feedbacks as proved in [13]. In that reference (see also [4]), it is also proposed the idea of hysteresis switching, leading to the class of dynamic hybrid controllers. In [14], the problem of uniting two output-feedback controllers is approached. The solution is provided as a hybrid controller, where the switching is performed by a norm observer. These results are extended in [15], considering the uniting of two hybrid output feedback controllers. All these previous works focus on uniting local and global controllers. However, it is well-known that there are systems which cannot be globally stabilized, for instance unstable linear systems with bounded control [16] and the examples in [10]. For those systems, instead of requiring controllers with global stability properties, we can aim at designing controllers for semi-global asymptotic stabilization. The work [19] shows that stabilizability and observability are sufficient conditions for semi-global stabilization by dynamic output feedback. This leads to the variation of the uniting problem presented in this work, where only local stability and attractivity properties are required. A similar problem has been studied in [5] in an input-output sense and under a state-independent input-to-output stability as- 
sumption. We consider two output feedback controllers, one (referred to as local controller) renders the system local asymptotic stable with some desired performance, and the other (referred to as regional controller) steers the trajectories starting from some given set to a neighborhood of the origin where the first controller applies. Clearly, the regional controller is assumed to have larger domain of attraction. The main goal of the proposed uniting problem is to enlarge the estimation of the domain of attraction of the local controller in a manner that the performance is not degraded on a neighborhood of the origin. The solution proposed consists of a switching logic between the controllers, which is implemented by a hybrid controller following the formalism for hybrid systems in [7]. As opposed to the results in $[14,15]$, our switching logic is based on a tunable observer. The assumption of a norm observer is weaker, but the rate of convergence of a norm observer is usually not tunable. As a consequence, the switching between the controllers may not be performed sufficiently fast to avoid a trajectory to leave the domain of attraction of the regional controller.

On the other hand, similarly to $[14,15]$, the robust local asymptotic stability of the proposed hybrid system is concluded by the hybrid basic conditions.

The outline of the paper is as follows. The uniting problem is introduced in Section 2. The main result follows in Section 3. First, a hybrid output feedback controller is designed, and second, the controller is proven to solve the uniting problem. Section 4 illustrates the effectiveness of the proposed solution by a numerical example.

Notation: Throughout this work, the following notation is used. The notation $\|x\|$ is the Euclidean norm for $x \in \mathbb{R}^{n}$. For a symmetric matrix $A \in \mathbb{R}^{n \times n}, \lambda_{m}(A)$ and $\lambda_{M}(A)$ stand for the minimum and maximum eigenvalue, respectively. A ball in $\mathbb{R}^{n}$ of radius $\varepsilon$ is denoted by $\mathbb{B}(\varepsilon):=\left\{x \in \mathbb{R}^{n}:\|x\| \leq \varepsilon\right\}$. The symbol $\ominus$ stands for the Minkowski difference. A function $f: \mathbb{R}_{+} \rightarrow$ $\mathbb{R}_{+}$is of class $\mathcal{K}$ if it is continuous, strictly increasing, and $f(0)=0$. The function $f$ is of class $\mathcal{K}_{\infty}$ if $f \in \mathcal{K}$ and $\lim _{s \rightarrow \infty} f(s)=\infty$. A continuous function $f: \mathbb{R}_{+} \times \mathbb{R}_{+} \rightarrow \mathbb{R}_{+}$is of class $\mathcal{K} \mathcal{L}$ if for each fixed $s$, the function $r \mapsto f(r, s)$ belongs to class $\mathcal{K}$ and for each fixed $r$, the function $s \mapsto f(r, s)$ is nonincreasing and $\lim _{s \rightarrow \infty} f(r, s)=0$. Given a set $S \subset \mathbb{R}^{n}$ and a point $x \in \mathbb{R}^{n},\|x\|_{S}:=\inf _{y \in S}\{\|x-y\|\}$. The reader is referred to [7] for the basic notation in hybrid systems.

\section{Problem statement}

Consider the following nonlinear systems defined by

$$
\dot{x}=f(x, u), y=h(x) \text {. }
$$

where $x \in \mathbb{R}^{n}$ is the state of the system, $u \in \mathbb{R}^{m}$ is the input, $y \in \mathbb{R}^{p}$ is the output, $f: \mathbb{R}^{n} \times \mathbb{R}^{m} \rightarrow \mathbb{R}^{n}$ is a locally
Lipschitz function with $f(0,0)=0$ and $h: \mathbb{R}^{n} \rightarrow \mathbb{R}^{p}$ is a continuously differentiable function with $h(0)=0$. In addition, let us consider two dynamic output feedback controllers, leading to the following closed-loop systems:

$$
\begin{aligned}
& \dot{x}=f\left(x, \alpha_{0}\left(\zeta_{0}, h(x)\right)\right), \\
& \dot{\zeta}_{0}=\varphi_{0}\left(\zeta_{0}, h(x)\right), \\
& \dot{x}=f\left(x, \alpha_{1}\left(\zeta_{1}, h(x)\right)\right), \\
& \dot{\zeta}_{1}=\varphi_{1}\left(\zeta_{1}, h(x)\right),
\end{aligned}
$$

where $\zeta_{q} \in \mathbb{R}^{l_{q}}$ and $\varphi_{q}: \mathbb{R}^{l_{q}} \times \mathbb{R}^{p} \rightarrow \mathbb{R}^{l_{q}}, \alpha_{q}: \mathbb{R}^{l_{q}} \times \mathbb{R}^{p} \rightarrow$ $\mathbb{R}^{m}, q \in\{0,1\}$, are continuous functions vanishing at the origin. The local controller defined by $\alpha_{0}$ and $\varphi_{0}$ is assumed to render the closed-loop system (2) locally asymptotically stable, while the regional controller given by $\alpha_{1}$ and $\varphi_{1}$ guarantees ultimate boundedness of the closed-loop system (3).

In this work, a hybrid output feedback controller is given by $(\mathcal{C}, \mathcal{D}, u, v, w)$, where $\mathcal{C} \subset \mathbb{R}^{l}$ and $\mathcal{D} \subset \mathbb{R}^{l}$ are closed sets and $u: \mathcal{C} \times \mathbb{R}^{p} \rightarrow \mathbb{R}^{m}, v: \mathcal{C} \times \mathbb{R}^{p} \rightarrow \mathbb{R}^{l}$, and $w:$ $\mathcal{D} \times \mathbb{R}^{p} \rightarrow \mathbb{R}^{l}$ are continuous functions. The closed-loop system that results from the feedback interconnection of the system (1) and the hybrid controller, is described under the hybrid systems framework of [7] as follows:

$$
\left.\begin{array}{l}
\dot{x}=f(x, u(\zeta, h(x)) \\
\dot{\zeta}=v(\zeta, h(x))
\end{array}\right\}, \zeta \in \mathcal{C},
$$

A solution to the hybrid system (4) consists of a $h y$ brid time domain $\operatorname{dom}(x, \zeta)$, and a hybrid arc $(x, \zeta)$ : $\operatorname{dom}(x, \zeta) \rightarrow \mathbb{R}^{n} \times \mathbb{R}^{l}$. The solutions are parametirezed by $(t, j)$, where $t$ is the ordinary time and $j$ corresponds to the number of jumps. Throughout this work, we will refer to the complete solutions to (4) simply as solutions. The reader is referred to [7] for more details about the hybrid system framework.

Let us recall that a compact set $\mathcal{A} \subset \mathbb{R}^{n} \times \mathbb{R}^{l}$ is locally asymptotically stable for system (4) if

- (stability) for all $\varepsilon>0$, there exists $\delta>0$ such that for all $\left(x^{0}, \zeta^{0}\right) \in \mathbb{R}^{n} \times(\mathcal{C} \cup \mathcal{D})$ satisfying $\left\|\left(x^{0}, \zeta^{0}\right)\right\|_{\mathcal{A}} \leq$ $\delta$, every trajectory to $(4)$ starting at $\left(x^{0}, \zeta^{0}\right)$ satisfies $\|(x(t, j), \zeta(t, j))\|_{\mathcal{A}} \leq \varepsilon$, for all $(t, j) \in \operatorname{dom}(x, \zeta)$;

- (attractivity) there exists $\delta_{a}>0$ such that for all $\left(x^{0}, \zeta^{0}\right) \in \mathbb{R}^{n} \times(\mathcal{C} \cup \mathcal{D})$ satisfying $\left\|\left(x^{0}, \zeta^{0}\right)\right\|_{\mathcal{A}} \leq \delta_{a}$, every trajectory to $(4)$ starting at $\left(x^{0}, \zeta^{0}\right)$ satisfies $\lim _{t+j \rightarrow \infty}\|(x(t, j), \zeta(t, j))\|_{\mathcal{A}}=0$. 
Let us assume that the domain of attraction of the closed-loop system (2) contains some set $\mathcal{B} \subset \mathbb{R}^{n} \times \mathbb{R}^{l_{0}}$. Thus, this work focuses on the following uniting problem:

Uniting problem: The problem is to find a hybrid output feedback controller $(\mathcal{C}, \mathcal{D}, u, v, w)$ such that

- there exist a matrix $M \in \mathbb{R}^{l_{0} \times l}$ and a compact set $\mathcal{A} \subset\{0\} \times \operatorname{ker}(M)$, such that the set $\mathcal{A}$ is locally asymptotically stable for the system (4) with a domain of attraction containing $\mathcal{B}_{\alpha}:=\left\{(x, \zeta) \in \mathbb{R}^{n} \times(\mathcal{C} \cup \mathcal{D})\right.$ : $\left.\frac{1}{\alpha}(x, M \zeta) \in \mathcal{B}\right\}$ for some $\alpha>1$;

- there exists a continuous positive definite function $\rho \in \mathbb{R}^{n} \times \mathbb{R}^{l} \rightarrow \mathbb{R}_{>0}$, and $r>0$ such that any trajectory of system (4) starting at $\left(x^{0}, \zeta^{0}\right)$, satisfying $\rho\left(x^{0}, \zeta^{0}\right) \leq r$, has the hybrid time domain $[0, \infty) \times\{0\}$ and $(x(t, 0), M \zeta(t, 0))=\left(\bar{x}(t), \bar{\zeta}_{0}(t)\right)$ for some trajectory $\left(\bar{x}, \bar{\zeta}_{0}\right)$ of $(2)$.

Roughly speaking, the uniting problem under study consists of two problems: first, by combining two different controllers, we look for an enlargement of the estimation $\mathcal{B}$ of the domain of attraction of the closed-loop system (2); second, there exists a projection from $\mathbb{R}^{n} \times \mathbb{R}^{l}$ to $\mathbb{R}^{n} \times \mathbb{R}^{l_{0}}$ such that the projected trajectories of the hybrid system (4) match the trajectories of the system (2) for small enough initial conditions.

Note that the set $\mathcal{B}_{\alpha}$ with $\alpha>1$ is defined in such a way that its projection onto the state-space of system (2) contains the estimation $\mathcal{B}$ of the domain of attraction of system (2). Therefore, if the domain of attraction of (4) contains $\mathcal{B}_{\alpha}$, then there are initial condition for which the solutions to (4) converges to $\mathcal{A}$, although the solutions to system (2) are not guaranteed to converge.

In order to provide an explicit solution of the uniting problem, let us state several assumptions. First, the local asymptotic stability of system (2) is formalized in terms of a Lyapunov function $V_{0}$. Similarly, we assume local attractivity for system (3) in terms of a Lyapunov function $V_{1}$. For the sake of clarity, two families of sets involving functions $V_{0}$ and $V_{1}$ are defined

$$
\begin{aligned}
& \Omega_{j}(\varepsilon):=\left\{\left(x, \zeta_{j}\right) \in \mathbb{R}^{n} \times \mathbb{R}^{l_{j}}: V_{j}\left(x, \zeta_{j}\right) \leq \varepsilon\right\}, \\
& \mathcal{P}_{j}(\varepsilon):=\left\{x \in \mathbb{R}^{n}: \exists \zeta_{j} \in \mathbb{R}^{l_{j}},\left(x, \zeta_{j}\right) \in \Omega_{j}(\varepsilon)\right\}, \\
& \mathcal{X}_{j}(\varepsilon):=\left\{x \in \mathbb{R}^{n}:(x, 0) \in \Omega_{j}(\varepsilon)\right\} .
\end{aligned}
$$

for $j \in\{0,1\}$. In other words, $\Omega_{j}(\varepsilon)$ is a Lyapunov set, $\mathcal{P}_{j}(\varepsilon)$ is its projection onto the $x$-component, and $\mathcal{X}_{j}(\varepsilon)$ is the intersection of Lyapunov level with the subspace $\mathbb{R}^{n}$.

Assumption 1 (Local asymptotic stability) There exist a continuously differentiable function $V_{0}: \mathbb{R}^{n} \times \mathbb{R}^{l_{0}} \rightarrow$

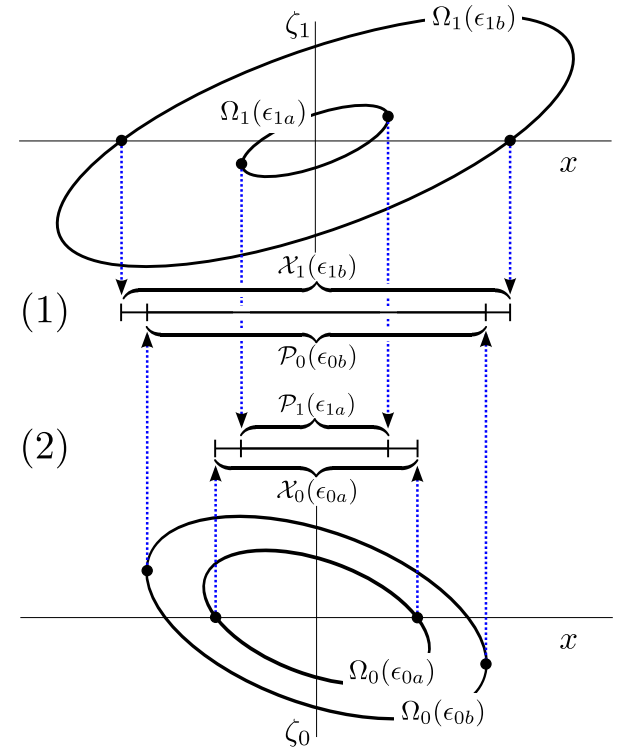

Fig. 1. Ilustration of Assumtion 3.

$\mathbb{R}_{\geq 0}$, functions $\underline{\alpha}_{0}, \bar{\alpha}_{0} \in \mathcal{K}_{\infty}$, and $\varepsilon_{0}>0$, such that for $\operatorname{all}\left(x, \zeta_{0}\right) \in \mathbb{R}^{n} \times \mathbb{R}^{l_{0}}$

$$
\begin{gathered}
\underline{\alpha}_{0}\left(\|x\|^{2}+\left\|\zeta_{0}\right\|^{2}\right) \leq V_{0}\left(x, \zeta_{0}\right) \leq \bar{\alpha}_{0}\left(\|x\|^{2}+\left\|\zeta_{0}\right\|^{2}\right) \\
\text { and for all }\left(x, \zeta_{0}\right) \in \Omega_{0}\left(\varepsilon_{0}\right) \backslash\{(0,0)\} \\
\left.\qquad \nabla V_{0}\left(x, \zeta_{0}\right),\left[f, \varphi_{0}\right]^{\top}\right\rangle<0 .
\end{gathered}
$$

Assumption 2 (Local attractivity and boundedness) There exist a continuous function $V_{1}: \mathbb{R}^{n} \times \mathbb{R}^{l_{1}} \rightarrow \mathbb{R}_{\geq 0}$, functions $\underline{\alpha}_{1}, \bar{\alpha}_{1} \in \mathcal{K}_{\infty}$, and $\varepsilon_{2} \geq \varepsilon_{1}>\varepsilon_{1 a}>0$, such that for all $\left(x, \zeta_{1}\right) \in \mathbb{R}^{n} \times \mathbb{R}^{l_{1}}$

$$
\underline{\alpha}_{1}\left(\|x\|^{2}+\left\|\zeta_{1}\right\|^{2}\right) \leq V_{1}\left(x, \zeta_{1}\right) \leq \bar{\alpha}_{1}\left(\|x\|^{2}+\left\|\zeta_{1}\right\|^{2}\right)
$$

and for all trajectories to (3) starting in $\Omega_{1}\left(\varepsilon_{1}\right)$, it follows $\lim \sup _{t \rightarrow \infty} V_{1}\left(x(t), \zeta_{1}(t)\right)<\varepsilon_{1 a}$, and $\left(x(t), \zeta_{1}(t)\right) \in \Omega_{1}\left(\varepsilon_{2}\right)$ for all $t \geq 0$.

The following assumption allows us to guarantee that when the system switches from dynamic (2) to (3), the system stays in the region of attractivity of system (3). In addition, it is assumed that all trajectories to system (3) starting in $\Omega_{1}\left(\varepsilon_{1}\right)$ tend to a set, which is contained in the domain of attraction of system (2) (see Fig. 1), as specified in the following assumption.

Assumption 3 There exist positive real numbers $\varepsilon_{0 a}$, $\varepsilon_{0 b}$, and $\varepsilon_{1 b}$, such that $\varepsilon_{0 a}<\varepsilon_{0 b}<\varepsilon_{0}, \varepsilon_{1 b}<\varepsilon_{1}$, and

(1) $\mathcal{P}_{0}\left(\varepsilon_{0 b}\right) \subset \mathcal{X}_{1}\left(\varepsilon_{1 b}\right)$,

(2) $\mathcal{P}_{1}\left(\varepsilon_{1 a}\right) \subset \mathcal{X}_{0}\left(\varepsilon_{0 a}\right)$.

Note that the local controller may be used with initial conditions for which the solutions to (2) are not stable. 
In these cases, it is necessary to switch to the regional controller before the solutions leave the estimation of the domain of attraction of the regional controller, so that the system can be stabilized. Since the proposed solution to the uniting problem consists on a switching law which depends on an estimation of the system state, it is required to guarantee that a small estimation error is obtained fast enough, that is, the convergence rate of the observer must be tuned, as it is precisely introduced in the following assumption.

Next, it is assumed that there exists a local observer for system (1), whose convergence rate can be tuned. This is necessary to design a switching law based on the Lyapunov function $V_{0}$. Note that the stability and attractivity properties of (2) and (3) are only local. Therefore, the observer is required to be tunable, and thus, it is guaranteed that a small estimation error is obtained fast enough, as it is precisely introduced in the following assumption.

Assumption 4 (Tunable observer) For any compact sets $\Omega_{x} \subset \mathbb{R}^{n}, \Omega_{o b s} \subset \Omega_{x}, \Omega_{u} \subset \mathbb{R}^{m}$, and for all $\varepsilon_{\text {obs }}>0, t_{\text {obs }}>0$, there exist a locally Lipschitz function $\psi: \mathbb{R}^{n} \times \mathbb{R}^{p} \times \mathbb{R}^{m} \rightarrow \mathbb{R}^{n}$ and a function $\beta \in \mathcal{K} \mathcal{L}$ such that for all initial conditions $x^{0} \in \Omega_{\text {obs }}$ and input $u: \mathbb{R}_{\geq} \rightarrow \Omega_{u}$, it is satisfied

1) $\left\|x(t)-\zeta_{\text {obs }}(t)\right\| \leq \beta\left(\left\|x^{0}-\zeta_{\text {obs }}^{0}\right\|, t\right), t \in\left[0, t_{\Omega_{x}}(x(\cdot))\right)$

2) if $t_{\Omega_{x}}(x(\cdot))>t_{\text {obs }}$ then $\left\|x(t)-\zeta_{o b s}(t)\right\| \leq \varepsilon_{o b s}$, $t \in\left[t_{o b s}, t_{\Omega_{x}}(x(\cdot))\right)$,

where $x(\cdot)$ is the solution to (1) with initial condition $x_{0}$ and input $u$, and $\zeta_{o b s}(\cdot)$ is the solution to

$$
\dot{\zeta}_{o b s}=\psi\left(\zeta_{o b s}, h(x), u\right)
$$

on $\left[0, t_{\Omega_{x}}(x(\cdot))\right)$ with initial condition $\zeta_{\text {obs }}^{0} \in \Omega_{\text {obs }}$. In addition, $t_{\Omega_{x}}(x(\cdot))$ is defined as the minimum time that the trajectory $x(\cdot)$ takes to leave the set $\Omega_{x}$, with $t_{\Omega_{x}}(x(\cdot))=$ $\infty$ if the trajectory does not leave the set.

Remark 1 For the sake of simplicity, we consider that the state of the observer is directly the estimation of the system state. However, the proposed solution can be straightforwardly adapted to the case in which the estimation of the system state is retrieved by a function of the observer state.

Remark 2 Assumption 4 simply refers to the existence of a local tunable observer as introduced in [3] (similar definition of a local tunable asymptotic observer has been used in [1] and [9]). In other words, there must exist a local observer characterized by equation (9) such that the estimation error, $\left\|x(t)-\zeta_{o b s}(t)\right\|$, is bounded and converges to zero for all trajectory $x(\cdot)$ does not leave the set $\Omega_{x}$. The observer is called tunable in the sense that its convergence rate can be tuned. An example of tunable observer is the well-known high-gain observer (see e.g. [6]), whose existence is guaranteed by assuming local uniform observability. Finally, note that small values of $t_{\text {obs }}$ may lead to a peaking phenomenon, but since the state estimation is not directly used in the feedback loop, it cannot make the system trajectory $x(\cdot)$ to leave the set $\Omega_{x}$. However, more switching betweens the controllers can be expected.

\section{Main results}

In this section, a hybrid output feedback controller is proposed as a combination of dynamics (2) and (3), that solves the uniting problem.

Consider the augmented state $\zeta=\left(\zeta_{0}, \zeta_{1}, \zeta_{\text {obs }}, q\right) \in \mathbb{R}^{l}$ with $l=l_{0}+l_{1}+n+1$, then the controller $(\mathcal{C}, \mathcal{D}, u, v, w)$ is defined by functions

$$
\begin{aligned}
& u: \mathcal{C} \times \mathbb{R}^{p} \rightarrow \mathbb{R}^{m} \\
&(\zeta, y) \mapsto \alpha_{q}\left(\zeta_{q}, y\right) \\
& v: \mathcal{C} \times \mathbb{R}^{p} \rightarrow \mathbb{R}^{l} \\
&(\zeta, y) \mapsto\left((1-q) \varphi_{0}\left(\zeta_{0}, y\right), q \varphi_{1}\left(\zeta_{1}, y\right), \varphi_{\text {obs }}(\zeta, y), 0\right) \\
& w: \mathcal{D} \times \mathbb{R}^{p} \rightarrow \mathbb{R}^{l} \\
&(\zeta, y) \mapsto\left(q \zeta_{0},(1-q) \zeta_{1}, \zeta_{\text {obs }}, 1-q\right)
\end{aligned}
$$

with $\varphi_{\text {obs }}(\zeta, y)=\psi\left(\zeta_{\text {obs }}, y, u(\zeta, y)\right)$ and sets, $\mathcal{C}=\mathcal{C}_{0} \cup \mathcal{C}_{1}$, $\mathcal{D}=\mathcal{D}_{0} \cup \mathcal{D}_{1}$, where

$$
\begin{aligned}
& \mathcal{C}_{0}=\left\{\zeta: V_{0}\left(\zeta_{o b s}, \zeta_{0}\right) \leq \varepsilon_{0 b}, \zeta_{1}=0, q=0\right\}, \\
& \mathcal{C}_{1}=\left\{\zeta: V_{0}\left(\zeta_{o b s}, \zeta_{0}\right) \geq \varepsilon_{0 a}, \zeta_{0}=0, q=1\right\}, \\
& \mathcal{D}_{0}=\left\{\zeta: V_{0}\left(\zeta_{o b s}, \zeta_{0}\right) \geq \varepsilon_{0 b}, \zeta_{1}=0, q=0\right\}, \\
& \mathcal{D}_{1}=\left\{\zeta: V_{0}\left(\zeta_{o b s}, \zeta_{0}\right) \leq \varepsilon_{0 a}, \zeta_{0}=0, q=1\right\}
\end{aligned}
$$

The real numbers $\varepsilon_{0 a}$ and $\varepsilon_{0 b}$ are as given in Assumptions 1,2 , and 3 . In addition, the function $\psi$ is given as in Assumption 4, where $\Omega_{x}=\mathcal{P}_{1}\left(\varepsilon_{2}\right)$, $\varepsilon_{o b s}$ is set such that $\mathrm{B}\left(\varepsilon_{\text {obs }}\right) \subset \operatorname{int}\left(\mathcal{X}_{1}\left(\varepsilon_{1}\right) \ominus \mathcal{X}_{1}\left(\varepsilon_{1 b}\right)\right)$. The real number $t_{\text {obs }}$, necessary to define $\psi$, is considered as a design parameter of the hybrid controller and depends on the particular control system setup. Beside $t_{o b s}$, let us consider a positive real number $\varepsilon_{I}<\varepsilon_{1}$, such that the set of initial conditions of the observer (9) is given by $\Omega_{o b s}=\mathcal{P}_{1}\left(\varepsilon_{I}\right)$.

Theorem 1 Suppose Assumptions 1, 2, 3, and 4 hold, and there exists a positive real number $\alpha>1$ such that $\mathcal{P}_{0}\left(\varepsilon_{0} \alpha\right) \subset \mathcal{X}_{1}\left(\varepsilon_{I}\right)$. Then, for every given $\delta_{0} \geq \bar{\alpha}_{0}^{-1}\left(\varepsilon_{0} \alpha\right)$ there exists $t_{\text {obs }}>0$ such that the hybrid output feedback controller (10)-(11) solves the uniting problem with $\mathcal{B}=$ $\Omega_{0}\left(\varepsilon_{0}\right)$ and $\mathcal{A}=\{0\}$ for all initial conditions $\left(x^{0}, \zeta^{0}\right) \in$ $\mathbb{R}^{n} \times \mathcal{C} \cup \mathcal{D}$ satisfying

$$
\left(x^{0}, \zeta_{1}^{0}\right) \in \Omega_{1}\left(\varepsilon_{I}\right),\left(\zeta_{o b s}^{0}, \zeta_{1}^{0}\right) \in \Omega_{1}\left(\varepsilon_{I}\right),\left\|\zeta_{0}^{0}\right\|^{2} \leq \delta_{0}
$$


Remark 3 The sets $\mathcal{C}_{q}$ and $\mathcal{D}_{q}$ are closed, and functions

$$
\begin{aligned}
(x, \zeta) & \mapsto(f(x, u(\zeta, h(x))), v(\zeta, h(x))), \\
(x, \zeta) & \mapsto w(\zeta, h(x))
\end{aligned}
$$

are continuous on $\mathbb{R}^{n} \times \mathcal{C}_{q}$ and $\mathbb{R}^{n} \times \mathcal{D}_{q}$, respectively. Therefore, the hybrid system (4) with (10) and (11) satisfies the basic hybrid conditions, and it follows from Proposition $6.30 \mathrm{in} \mathrm{[7],} \mathrm{that} \mathrm{the} \mathrm{system} \mathrm{is} \mathrm{well-posed.} \mathrm{As} \mathrm{a} \mathrm{con-}$ sequence, if the hypothesis of Theorem 1 hold, then the hybrid system (4) with (10) and (11) is also robustly locally asymptotically stable by Theorem 7.21 in [7]. Note that the considered robust asymptotic stability means, roughly speaking, that there exists a sufficiently small perturbation (disturbance, uncertainty, noise, etc) for which the the hybrid system remains asymptotically stable. However, its behavior and performance may be completely different for such small perturbation. This fact may happen when a high-gain observer is used, due to the trade-off between the speed of convergence of the estimation error and the sensitivity to measurement noise.

PROOF. First, let us conclude some basic properties of the solutions. As it is commented in Remark 3, the hybrid system (4) with (10) and (11) satisfied the basic hybrid conditions. In addition, from the definition of $\mathcal{C}$ and $\mathcal{D}$, we get $\partial \mathcal{C} \backslash \mathcal{D}=\emptyset$, which implies, $\zeta \in \operatorname{int} \mathcal{C}$ for all $\zeta \in \mathcal{C} \backslash \mathcal{D}$, and $T_{\mathcal{C}}(\zeta)=\mathbb{R}^{l}$, where $T_{\mathcal{C}}(\zeta)$ stands for the tangent cone to $\mathcal{C}$ at $\zeta$. Therefore, it directly follows $\{v(\zeta, y)\} \in T_{\mathcal{C}}(\zeta)$, and the viability conditions of Proposition 6.10 in [7] are satisfied. Since $w(\zeta, y) \in$ $(\mathcal{C} \cup \mathcal{D})$, then for all initial conditions $\left(x^{0}, \zeta^{0}\right) \in \mathbb{R}^{n} \times(\mathcal{C} \cup$ $\mathcal{D})$, there exists a maximal trajectory to $(4)$ starting at $\left(x^{0}, \zeta^{0}\right)$. In addition, every trajectory has an unbounded hybrid time domain or eventually leaves any compact subset of $\mathbb{R}^{n} \times \mathbb{R}^{l}$.

Stability: For a given $\varepsilon>0$, consider an arbitrarily $t_{o b s}>0$ and a positive $\bar{\varepsilon} \leq \bar{\alpha}_{0}^{-1}\left(\varepsilon_{0 a}\right)$, then for all $\left(\zeta_{\text {obs }}, \zeta_{0}\right)$ satisfying $\left\|\zeta_{0}\right\|^{2}+\left\|\bar{\zeta}_{\text {obs }}\right\|^{2} \leq \bar{\varepsilon}$, it follows $\left(\zeta_{o b s}, \zeta_{0}\right) \in \Omega_{0}\left(\varepsilon_{0 a}\right)$ by inequality (6). Let us define $\varrho(s)=3\left(\underline{\alpha}_{0}^{-1}\left(\bar{\alpha}_{0}(s)\right)+\beta(2 \sqrt{s}, 0)\right)$. From the properties of $\alpha_{0}, \bar{\alpha}_{0}$, and $\beta$, it follows that $\varrho \in \mathcal{K}$. Now we assume without loss of generality that $0<\varepsilon \leq \bar{\varepsilon}$. Let us pick $\delta$ such that $0<\delta<\min \left(1, \varrho^{-1}(\varepsilon)\right)$ then from Assumptions 1 and 4 for all initial conditions $\left(x^{0}, \zeta_{0}^{0}, \zeta_{\text {obs }}^{0}\right)$ satisfying $\left\|x^{0}\right\|^{2}+\left\|\zeta_{0}^{0}\right\|^{2}+\left\|\zeta_{o b s}^{0}\right\|^{2} \leq \delta$ the trajectory to $(2)$ and (9) with $u=\alpha_{0}\left(\zeta_{0}, h(x)\right)$, starting at $\left(x^{0}, \zeta_{0}^{0}, \zeta_{o b s}^{0}\right)$, satisfies

$$
\|x(t)\|^{2}+\left\|\zeta_{0}(t)\right\|^{2}+\left\|\zeta_{o b s}(t)\right\|^{2} \leq \varepsilon
$$

for all $t \geq 0$.

Now consider a trajectory to (4) with initial condition $\left(x^{0}, \zeta^{0}\right) \in \mathbb{R}^{n} \times(\mathcal{C} \cup \mathcal{D})$ such that $\left\|x^{0}\right\|^{2}+\left\|\zeta_{0}^{0}\right\|^{2}+$ $\left\|\zeta_{\text {obs }}^{0}\right\|^{2}+q^{0} \leq \delta$. Since $\delta<1$, it follows $q^{0}=0$. In addition, $\left(\zeta_{o b s}^{0}, \zeta_{0}^{0}\right) \in \Omega_{0}\left(\varepsilon_{0 a}\right)$ holds, and thus, the initial condition is in $\mathcal{C}_{0}$. Since $\varepsilon_{0 a}<\varepsilon_{0 b}$, there exists a $T \in \mathbb{R}_{>0} \cup\{\infty\}$ such that the components $x(\cdot, 0), \zeta_{0}(\cdot, 0)$ and $\zeta_{o b s}(\cdot, 0)$ of the trajectory $(x, \zeta)$ are also a trajectory to (2) and (9) with $u=\alpha_{0}\left(\zeta_{0}, h(x)\right)$ on $[0, T)$. In addition, condition (14) and $\varepsilon \leq \bar{\varepsilon} \leq \bar{\alpha}_{0}^{-1}\left(\varepsilon_{0 a}\right)$ imply $\left(\zeta_{o b s}(t, 0), \zeta_{0}(t, 0)\right) \in \Omega_{0}\left(\varepsilon_{0 a}\right)$ for all $t \in[0, T)$. Therefore, from the expression of $\mathcal{C}$, all such maximal trajectories do not reach $\mathcal{D}$, and their domains are of the form $[0, \infty) \times\{0\}$. Since $\zeta_{1}(t, 0)=0, q(t, 0)=0$, and $\|x(t, 0)\|^{2}+\left\|\zeta_{0}(t, 0)\right\|^{2}+\left\|\zeta_{\text {obs }}(t, 0)\right\|^{2} \leq \varepsilon$ for all $t \geq 0$, the stability is proved.

Moreover, every trajectory to (4) starting at $\left(x^{0}, \zeta^{0}\right) \in$ $\mathbb{R}^{n} \times(\mathcal{C} \cup \mathcal{D})$ such that $\rho\left(x^{0}, \zeta^{0}\right)=\left\|x^{0}\right\|^{2}+\left\|\zeta_{0}^{0}\right\|^{2}+$ $\left\|\zeta_{o b s}^{0}\right\|^{2}+q^{0} \leq r=\delta$, has the hybrid time domain $[0, \infty) \times$ $\{0\}$. In addition, $(x(t, 0), M \zeta(t, 0))=\left(\bar{x}(t), \bar{\zeta}_{0}(t)\right)$ with $M=\left[\begin{array}{llll}I & 0 & 0 & 0\end{array}\right]$, where $\left(\bar{x}, \bar{\zeta}_{0}\right)$ is a trajectory to $(2)$. Therefore, the second statement of the uniting problem holds.

To conclude the proof of Theorem 1, it remains to guarantee the attractivity of system (4).

Attractivity: Let $(x, \zeta)$ be a maximal trajectory to $(4)$ starting at $\left(x^{0}, \zeta^{0}\right)$ satisfying (12). For the sake of clarity, the proof is divided into 4 claims:

First, it is proved that if the trajectory leaves the set $\Omega_{1}\left(\varepsilon_{1}\right)$, then the discrete variable switches to 1 before leaving the set. This fact, together with Assumption 2, implies that the trajectory returns to $\Omega_{1}\left(\varepsilon_{1}\right)$ eventually.

Claim 1: There does not exist $(\bar{t}, \bar{j}) \in \operatorname{dom}(x, \zeta)$, such that $q(\bar{t}, \bar{j})=0$ and $V_{1}\left(x(\bar{t}, \bar{j}), \zeta_{1}(\bar{t}, \bar{j})\right) \geq \varepsilon_{1}$.

Proof of Claim 1. Let us prove the claim by contradiction. Suppose there is $(\bar{t}, \bar{j}) \in \operatorname{dom}(x, \zeta)$ such that $V_{1}\left(x(\bar{t}, \bar{j}), \zeta_{1}(\bar{t}, \bar{j})\right)=\varepsilon_{1}$ and $q(\bar{t}, \bar{j})=0$. Note that $\left(x^{0}, \zeta_{1}^{0}\right) \in \mathcal{P}_{1}\left(\varepsilon_{I}\right)$ implies $V_{1}\left(x^{0}, \zeta_{1}^{0}\right) \leq \varepsilon_{I}<\varepsilon_{1}$. Since the set of initial conditions is bounded, as stated in (12), the rate of divergence is also bounded for a small enough interval of time. Therefore, there exists $t_{o b s}>0$ independent of the trajectory, such that $\bar{t}>t_{\text {obs }}$. That is, $t_{o b s}$ is the minimum time that a trajectory may take to reach the $\varepsilon_{1}$-level of the function $V_{1}$. In addition, from the expression of $\mathcal{C}_{0}, \mathcal{D}_{0}$ and the function $w$, it is obtained

$$
\left(\zeta_{o b s}(\bar{t}, \bar{j}), \zeta_{0}(\bar{t}, \bar{j})\right) \in \Omega_{0}\left(\varepsilon_{0 b}\right) .
$$

Assumption 4 leads to $x(\bar{t}, \bar{j})-\zeta_{o b s}(\bar{t}, \bar{j}) \in \mathbb{B}\left(\varepsilon_{o b s}\right)$. Item 1 of Assumption 3 and equation (15) imply $\zeta_{o b s}(\bar{t}, \bar{j}) \in \mathcal{X}_{1}\left(\varepsilon_{1 b}\right)$. In addition, since $\varepsilon_{o b s}$ is chosen such that $\mathbb{B}\left(\varepsilon_{o b s}\right) \subset \operatorname{int}\left(\mathcal{X}_{1}\left(\varepsilon_{1}\right) \ominus \mathcal{X}_{1}\left(\varepsilon_{1 b}\right)\right)$, it is obtained $x(\bar{t}, \bar{j}) \in \operatorname{int}\left(\mathcal{X}_{1}\left(\varepsilon_{1}\right)\right)$, which contradicts $V_{1}\left(x(\bar{t}, \bar{j}), \zeta_{1}(\bar{t}, \bar{j})\right)=\varepsilon_{1}\left(\right.$ note that $\left.\zeta_{1}(\bar{t}, \bar{j})=0\right)$.

Now, it is proved that the discrete variable is 0 for some suitable hybrid time. 
Claim 2: For all initial conditions $\left(x^{0}, \zeta^{0}\right)$ satisfying $\left(x^{0}, \zeta_{1}^{0}\right) \in \Omega_{1}\left(\varepsilon_{1}\right)$, there exists $(t, j) \in \operatorname{dom}(x, \zeta)$, such that $q(t, j)=0$.

Proof of Claim 2. By way of contradiction, let assume that

$$
q(t, j)=1, \forall(t, j) \in \operatorname{dom}(x, \zeta) .
$$

Due to the expression of $\mathcal{C}_{1}, \mathcal{D}_{1}$ and the function $w$, it follows that $\operatorname{dom}(x, \zeta)=[0, T) \times\{0\}$ for some $T \in$ $\mathbb{R}_{>0} \cup\{\infty\}$. Note that the components $x(\cdot, 0), \zeta_{1}(\cdot, 0)$ and $\zeta_{\text {obs }}(\cdot, 0)$ of the trajectory $(x, \zeta)$ are also a trajectory to (3) and (9) with $u=\alpha_{1}\left(\zeta_{1}, h(x)\right)$ on $[0, T)$. From Assumption 2 , every trajectory to $(3)$ starting at $\left(x^{0}, \zeta_{1}^{0}\right) \in$ $\Omega_{1}\left(\varepsilon_{1}\right)$ exists on $[0, \infty)$ and $\left(x(t), \zeta_{1}(t)\right) \in \Omega_{1}\left(\varepsilon_{2}\right)$ for all $t \geq 0$. In addition, the set $\Omega_{x}$ in Assumption 4 is given by $\Omega_{x}=\mathcal{P}_{1}\left(\varepsilon_{2}\right)$, and thus Assumption 4 guarantees that the solution, $\zeta_{o b s}$, to $(9)$ exists on $[0, \infty)$ for all initial conditions $\zeta_{\text {obs }}^{0} \in \mathcal{X}_{\text {obs }}$. The expression of $\mathcal{C}_{1}$ implies $\zeta_{0}(t, 0)=0$ for all $t \in[0, T)$. Thus, it follows that $T=\infty$.

Now, according to Assumption 2, there exists $\bar{t}>0$, such that $V_{1}\left(x(\bar{t}, 0), \zeta_{1}(\bar{t}, 0)\right)<\varepsilon_{1 a}$. In addition, from Assumption $4, \bar{t}$ can be chosen sufficiently large such that $\zeta_{\text {obs }}(\bar{t}, 0) \in \mathcal{P}_{1}\left(\varepsilon_{1 a}\right)$. Considering item 2 of Assumption 3 , it is obtained $\zeta_{o b s}(\bar{t}, 0) \in \mathcal{X}_{0}\left(\varepsilon_{0 a}\right)$. In addition, $\left(\zeta_{\text {obs }}(\bar{t}, 0), \zeta_{0}(\bar{t}, 0)\right) \in \Omega_{0}\left(\varepsilon_{0 a}\right)$ since $\zeta_{0}(\bar{t}, 0)=0$. This contradicts (16), and concludes the proof of Claim 2.

Next, it is proved that if the discrete variable remains 0 after some sufficiently large hybrid time, then the trajectory converges to the origin.

Claim 3: If there exists a hybrid time $(\bar{t}, \bar{j}) \in \operatorname{dom}(x, \zeta)$, such that $q(t, j)=0$, for all $(t, j) \in \operatorname{dom}(x, \zeta),(\bar{t}, \bar{j}) \geq$ $(t, j)$, then the trajectory is complete and converges to the origin.

Proof of Claim 3. First note that there is no jump after $(\bar{t}, \bar{j})$, then for all $(t, j) \in \operatorname{dom}(x, \zeta),(\bar{t}, \bar{j}) \geq(t, j)$ it follows $j=\bar{j}$ and $(t, \bar{j}) \in \operatorname{dom}(x, \zeta)$ for all $t \geq \bar{t}$. In addition, $\left(x(\cdot, \bar{j}), \zeta_{0}(\cdot, \bar{j})\right)$ and $\zeta_{o b s}(\cdot, \bar{j})$ are trajectories to (2) and (9) with $u=\alpha_{0}\left(\zeta_{0}, h(x)\right)$, respectively, on the domain $\{t:(t, \bar{j}) \in \operatorname{dom}(x, \zeta)\}$.

Let us now prove that the trajectory is complete. First, we have $\zeta_{1}(t, \bar{j})=0$ for all $(t, \bar{j}) \in \operatorname{dom}(x, \zeta), t \geq \bar{t}$, and $\left(\zeta_{0}(t, \bar{j}), \zeta_{\text {obs }}(t, \bar{j})\right)$ is bounded due to the expression of $\mathcal{C}_{0}$. In addition, Claim 1 implies $\left(x(t, \bar{j}), \zeta_{1}(t, \bar{j})\right) \in$ $\Omega_{1}\left(\varepsilon_{1}\right)$ for all $(t, \bar{j}) \in \operatorname{dom}(x, \zeta)$, and thus $x(t, \bar{j})$ is also bounded. From the boundedness of $(x, \zeta)$ and Proposition 6.10 of [7], it follows that the trajectory is complete.

The components $\left(x(t, \bar{j}), \zeta_{0}(t, \bar{j})\right)$ and $\zeta_{\text {obs }}(t, \bar{j})$ with $u=$ $\alpha_{0}\left(\zeta_{0}, h(x)\right)$ are also trajectories to $(2)$ and $(9)$, respectively, on $t \in[\bar{t}, \infty)$, and thus, from Assumption 4, $\varepsilon_{0 b}<\varepsilon_{0}$, and the fact that $\beta$ is a function of class $\mathcal{K} \mathcal{L}, \bar{t}$ may be assumed to be sufficiently large such that $\left(x(t, \bar{j}), \zeta_{0}(t, \bar{j})\right) \in \Omega_{0}\left(\varepsilon_{0}\right)$ for all $t \in[\bar{t}, \infty)$. According to
Assumption 1, this set belongs to the domain of attraction of $(2)$, and thus, $\left(x, \zeta_{0}\right)$ converges to the origin. The convergence of $\zeta_{o b s}$ is guaranteed by Assumption 4 .

The last claim proves that the discrete variable cannot switch back and forth between $q=0$ and $q=1$ :

Claim 4: There does not exist a non-decreasing sequence of hybrid times $\left(\left(t_{n}, j_{n}\right)_{n \in \mathbb{N}}\right) \in \operatorname{dom}(x, \zeta)$, such that we have, for all $n \in \mathbb{N}$,

$$
q\left(t_{2 n}, j_{2 n}\right)=0, q\left(t_{2 n+1}, j_{2 n+1}\right)=1 \text {. }
$$

Proof of Claim 4. First, it should be pointed out that instantaneous Zeno solutions are not possible due to the expressions of $\mathcal{D}_{0}$ and $\mathcal{D}_{1}$. First, let us prove the following intermediate result:

$$
\left(x(t, j), \zeta_{1}(t, j)\right) \in \Omega_{1}\left(\varepsilon_{2}\right), \forall(t, j) \in \operatorname{dom}(x, \zeta)
$$

Consider a trajectory to system (4) that leaves the set $\Omega_{1}\left(\varepsilon_{1}\right)$ at hybrid time $(\bar{t}, \bar{j}) \in \operatorname{dom}(x, \zeta)$, then the components $x(\cdot, \bar{j})$, and $\zeta_{1}(\cdot, \bar{j})$ of the trajectory $(x, \zeta)$ are also a trajectory to (3). Note that Claim 1 assures that $q$ does not switch to 0 until the trajectory does not return to the set $\Omega_{1}\left(\varepsilon_{1}\right)$. Finally, Assumption 2 guarantees that the trajectory does not leave the set $\Omega_{1}\left(\varepsilon_{2}\right)$ as stated in (18). A direct consequence of the above result is that items 1 and 2 of Assumption 4 hold for all $(t, j) \in \operatorname{dom}(x, \zeta)$.

Now, Claim 3 is proved by way of contradiction. Let assume that there exists a non-decreasing sequence of hybrid times $\left(\left(t_{n}, j_{n}\right)_{n \in \mathbb{N}}\right) \in \operatorname{dom}(x, \zeta)$, such that $(17)$ holds for all $n \in \mathbb{N}$. Without loss of generality, it is assumed that there is no jumps between two points of the sequence and that $j_{n}=n$. Due to the expression of the function $w$ and the $\operatorname{sets} \mathcal{C}$ and $\mathcal{D}$, for all $n \in \mathbb{N}$, the system flows in $\mathcal{C}_{0}$ between $\left(t_{2 n}, 2 n\right)$ and $\left(t_{2 n+1}, 2 n\right)$, and flows in $\mathcal{C}_{1}$ between $\left(t_{2 n+1}, 2 n+1\right)$ and $\left(t_{2 n+2}, 2 n+1\right)$. Hence, the definition of the sets $\mathcal{C}_{0}$ and $\mathcal{C}_{1}$ and the inequality $\varepsilon_{0 a}<\varepsilon_{0 b}$ imply that $\lim _{n \rightarrow \infty} t_{2 n}=\infty$. In addition, note that $\zeta\left(t_{2 n+2}, 2 n+1\right) \in \mathcal{D}_{1}$, and thus, $V_{0}\left(\zeta_{o b s}\left(t_{2 n+2}, 2 n+\right.\right.$ $\left.1), \zeta_{0}\left(t_{2 n+2}, 2 n+1\right)\right) \leq \varepsilon_{0 a}$, for all $n \in \mathbb{N}$. Therefore, from item 2 of Assumption 4 and the continuity of $V_{0}$, for a given $\eta>0$ there exists a sufficiently large integer $N>0$ (from Assumption 4 the estimation error of the observer can be reduced by increasing $N$ ) such that

$$
\begin{aligned}
& V_{0}\left(x\left(t_{2 N+2}, 2 N+1\right), \zeta_{0}\left(t_{2 N+2}, 2 N+1\right)\right) \\
& \leq V_{0}\left(\zeta_{o b s}\left(t_{2 N+2}, 2 N+1\right), \zeta_{0}\left(t_{2 N+2}, 2 N+1\right)\right)+\eta
\end{aligned}
$$

$$
\begin{aligned}
& V_{0}\left(\zeta_{o b s}\left(t_{2 N+2}, 2 N+1\right), \zeta_{0}\left(t_{2 N+2}, 2 N+1\right)\right) \\
& \leq V_{0}\left(x\left(t_{2 N+2}, 2 N+1\right), \zeta_{0}\left(t_{2 N+2}, 2 N+1\right)\right)+\eta
\end{aligned}
$$


Considering $\eta<0.5\left(\varepsilon_{0 b}-\varepsilon_{0 a}\right)$, it follows

$V_{0}\left(x\left(t_{2 N+2}, 2 N+1\right), \zeta_{0}\left(t_{2 N+2}, 2 N+1\right)\right) \leq \varepsilon_{0 a}+\eta<\varepsilon_{0}$.

Thus, between $\left(t_{2 N+2}, 2 N+2\right)$ and $\left(t_{2 N+3}, 2 N+2\right)$, $\left(x, \zeta_{0}\right)$ is a trajectory to $(2)$ starting in $\Omega_{0}\left(\varepsilon_{0}\right)$. In addition, equations (7) and (20) imply $V_{0}\left(\zeta_{o b s}\left(t_{2 n+3}, 2 n+\right.\right.$ $\left.2), \zeta_{0}\left(t_{2 n+3}, 2 n+2\right)\right) \leq \varepsilon_{0 a}+\eta<\varepsilon_{0 b}$. This contradicts $\zeta\left(t_{2 N+3}, 2 n+2\right) \in \mathcal{D}_{0}$ and concludes the proof of Claim 4 .

Let us conclude the proof combining the previous claims. First, pick an initial condition satisfying (12), then Claim 2 applies, and thus, there exist $\left(t_{1}, j_{1}\right) \in \operatorname{dom}(x, \zeta)$, such that, $q\left(t_{1}, j_{1}\right)=0$. In addition, Claim 1 guarantees that if the trajectory eventually leaves the set $\Omega_{1}\left(\varepsilon_{1}\right)$ then there exist $\left(t_{2}, j_{2}\right) \in \operatorname{dom}(x, \zeta),\left(t_{2}, j_{2}\right)>\left(t_{1}, j_{1}\right)$ such that, $q\left(t_{2}, j_{2}\right)=1$ and $V_{1}\left(x\left(t_{2}, j_{2}\right), \zeta_{1}\left(t_{2}, j_{2}\right)\right)=\varepsilon_{1}$. Hence, the trajectories are bounded, and in addition, Assumption 2 implies the trajectory will return to $\Omega_{1}\left(\varepsilon_{1}\right)$. By using again Claim 2 , there exist $\left(t_{3}, j_{3}\right) \in \operatorname{dom}(x, \zeta)$ $\left(t_{3}, j_{3}\right)>\left(t_{2}, j_{2}\right)$, such that, $q\left(t_{3}, j_{3}\right)=0$. Whether the trajectory remains in $\Omega_{1}\left(\varepsilon_{1}\right)$ or the above reasoning is repeated, Claim 4 assures that there exists a finite number switching, and thus, Claim 3 applies. Therefore, the trajectory is complete and tends to the origin. The stability and the attractivity imply the local asymptotic stability of the system. The enlargement of the estimation of the domain of attraction comes directly from $\mathcal{P}_{0}\left(\varepsilon_{0} \alpha\right) \subset \mathcal{X}_{1}\left(\varepsilon_{I}\right)$ and $\delta_{0} \geq \bar{\alpha}_{0}^{-1}\left(\varepsilon_{0} \alpha\right)$ as stated in the Theorem.

\section{Example}

In this section, a numerical example is provided in order to illustrate the interest of the proposed hybrid controller. Consider a nonlinear system (1) with $f$ and $h$ given by

$$
f(x, u)=f_{n}(x)+A x+B \operatorname{sat}(u), h(x)=C x
$$

with the state $x=\left[x_{1}, x_{2}, x_{3}\right]^{\top} \in \mathbb{R}^{3}$, a nonlinearity $f_{n}(x)=\left[0, x_{1}^{2}-2 x_{1} x_{3}, 0\right]^{\top}$ and matrices

$$
A=\left[\begin{array}{ccc}
-1 & 0 & 1 \\
0 & -1 & 1 \\
0 & -1 & 0
\end{array}\right], B=\left[\begin{array}{l}
0 \\
0 \\
1
\end{array}\right], C=\left[\begin{array}{lll}
1 & 0 & 0
\end{array}\right]
$$

The control signal ${ }^{1}$ is affected by a symmetric saturation defined by

$$
\operatorname{sat}(u)=\left\{\begin{aligned}
-\bar{u} & \text { if } \quad u<-\bar{u} \\
u & \text { if }-\bar{u} \leq u \leq \bar{u} \\
\bar{u} & \text { if } \quad \bar{u}<u
\end{aligned}\right.
$$

where $\bar{u}>0$ is the level of saturation. As a control objective, it is considered the local asymptotical stabilization of the system, and the local minimization of the cost function

$$
J(x, u)=\int_{0}^{\infty} x(s)^{\top} Q x(s)+R u(s)^{2} d s,
$$

where $Q \in \mathbb{R}^{n \times n}$ is a positive definite matrix and $R$ is a positive real number. A linear approximation of (1) with $f$ and $h$ defined in (22) is simply obtained by setting $f_{n}(x)=0$ and neglecting the saturation. Thus, it is considered an optimal output feedback control for the linear system, given by

$$
\varphi_{0}\left(\zeta_{0}, y\right)=(A-B K-L C) \zeta_{0}+L C y, \alpha_{0}\left(\zeta_{0}, y\right)=K \zeta_{0}
$$

where $\zeta_{0} \in \mathbb{R}^{3}, K=R^{-1} B^{\top} P_{K}$ and $L=R_{2}^{-1} P_{L} C^{\top}$ and matrices $P_{K}$ and $P_{L}$ are the solutions of the following algebraic Riccati equations

$$
\begin{aligned}
& A^{\top} P_{K}+P_{K} A-P_{K} B^{\top} R^{-1} B P_{K}+Q=0, \\
& A P_{L}+P_{L} A^{\top}-P_{L} C^{\top} R_{e}^{-1} C P_{L}+Q_{e}=0 .
\end{aligned}
$$

Matrix $Q_{e} \in \mathbb{R}^{n \times n}$ is positive definite and $R_{e}$ is a positive real number. They define the observer cost function:

$$
\int_{0}^{\infty}\left(x(s)-\zeta_{0}(s)\right)^{\top}\left(Q_{e}+L R_{e} L^{\top}\right)\left(x(s)-\zeta_{0}(s)\right) d s .
$$

Now consider the Lyapunov function $V_{0}\left(x, \zeta_{0}\right)=$ $\left[x^{\top}, \zeta_{0}^{\top}\right] P\left[x^{\top}, \zeta_{0}^{\top}\right]^{\top}$, and $\varepsilon_{0}=1$, then there exists a positive definite matrix $P$ such that $\Omega_{0}\left(\varepsilon_{0}\right)$ is in the nonsaturated region and inside the domain of attraction of system (2). It can be checked that $\left\|f_{n}(x)\right\|^{2} \leq k\left(x_{1}^{2}+x_{3}^{2}\right)$ holds for all $k>0$ and $x_{1}$ and $x_{3}$ with $\left|x_{1}\right|<\sqrt{k / 5}$. Therefore, it is possible to set an optimization problem to maximize the estimation of the domain of attraction. The details are omitted due to the space limitation.

On the other hand, suppose $|u| \leq \bar{u}$ and consider the Lyapunov function $V_{1}(x)=x_{1}^{2}+\left(x_{1}^{2}+x_{2}\right)^{2}+x_{3}^{2}$, then it follows

$\nabla V_{1}(x) \cdot f(x, u)=-2 x_{1}^{2}-2\left(x_{1}^{2}+x_{2}\right)^{2}+2 x_{1}^{2} x_{3}+2 x_{1} x_{3}+2 x_{3} u$.

\footnotetext{
${ }^{1}$ It can be proved that there does not exist a control signal that globally stabilizes the system.
} 
The control law $\alpha_{1}(y)=-y^{2}-y$ implies $\nabla V_{1}(x)$. $f\left(x, \alpha_{1}(h(x))\right)=-2 x_{1}^{2}-2\left(x_{1}^{2}+x_{2}\right)^{2}$. In addition, for a given $\bar{u}>0$, if $\varepsilon_{1}>0$ satisfies $\varepsilon_{1}+\sqrt{\varepsilon_{1}}=\bar{u}$, then $\alpha_{1}(h(x)) \leq \bar{u}$ for all $x \in \Omega\left(\varepsilon_{1}\right)$. By using LaSalle's invariance theorem (see for instance [8]), it is proved that system (3) with $l_{1}=0$ and $\alpha_{1}(y)=-y^{2}-y$ is locally asymptotically stable with domain of attraction containing the set $\Omega_{1}\left(\varepsilon_{1}\right)$.

To guarantee Assumption 4, it is considered a high-gain observer given by

$$
\psi_{o b s}\left(\zeta_{o b s}, y, u\right)=f\left(\zeta_{o b s}, u\right)+\Delta_{\theta} K\left(h\left(\zeta_{o b s}\right)-y\right),
$$

where

$$
\Delta_{\theta}=\left[\begin{array}{ccc}
\theta & 0 & 0 \\
0 & \theta^{2} & 0 \\
0 & 0 & \theta^{3}
\end{array}\right], K=\left[\begin{array}{l}
-3 \\
-3 \\
-1
\end{array}\right] .
$$

The observer gain $\theta>0$ should be sufficiently large to guarantee the convergence of the estimation error. In addition, it follows that for some constant $c>0$ (local Lipschitz constant of the nonlinearity of the system in the observable canonical form) (see e.g. [3])

$$
\left\|x(t)-\zeta_{o b s}(t)\right\|^{2} \leq \frac{\lambda_{M}\left(P_{o}\right)}{\lambda_{m}\left(P_{o}\right)} e^{-\left(\theta-2 \lambda_{M}\left(P_{o}\right) c\right) t}\left\|x^{0}-\zeta_{o b s}^{0}\right\|^{2},
$$

where the symmetric matrix $P_{o}$ is the solution of

$$
\left(\left[\begin{array}{lll}
0 & 1 & 0 \\
0 & 0 & 1 \\
0 & 0 & 0
\end{array}\right]+K C\right)^{\top} P_{o}+P_{o}\left(\left[\begin{array}{lll}
0 & 1 & 0 \\
0 & 0 & 1 \\
0 & 0 & 0
\end{array}\right]+K C\right)+I=0 .
$$

For the numerical illustration, suppose $R=20, R_{e}=$ $0.001, Q_{e}=I$ and matrix $Q$ is given by

$$
Q=\left[\begin{array}{ccc}
2 & 0 & -1 \\
0 & 1 & 0 \\
-1 & 0 & 2
\end{array}\right]
$$

The matrix $Q$ is chosen to minimize the effect of the nonlinearity $f_{n}(x)$, so it may obtain larger estimation of the domain of attraction of system (2). The saturation level is $\bar{u}=10$, and thus, $\varepsilon_{1}=7.29$. Matrices $P_{K}$ and $P_{L}$ are obtained by solving the Riccati equations with Matlab. Matrix $P$ is then obtained by solving BMIs (Bilinear Matrix Inequalities). Note that the origin of system (3) is locally asymptotically stable and thus $\varepsilon_{1 a}$ in Assumption 2 and Assumption 3 can chosen arbitrarily small. In this way, Assumption 4 is satisfied by setting $\varepsilon_{0 a}=0.81, \varepsilon_{0 b}=0.9$, and $\varepsilon_{1 b}=2.04$. In addition, the domain of attraction of system (3) contains $\Omega_{1}\left(\varepsilon_{1}\right)$, then it can be set $\varepsilon_{2}=\varepsilon_{1}$.

On the other hand, let $\varepsilon_{I}=5.83$, then, considering the saturation level and $\nabla V_{1}(x) \cdot f(x, u)$, it can be seen that
0.0255 seconds is a lower bound of the minimum time that it is necessary for the hybrid system to go from $V_{1}(x)=\varepsilon_{I}$ to $V_{1}(x)=\varepsilon_{1}$. Hence, it is necessary to find $\theta$ and $\varepsilon_{o b s}$ such that $\mathrm{B}\left(\varepsilon_{o b s}\right) \subset \operatorname{int}\left(\mathcal{X}_{1}\left(\varepsilon_{1}\right) \ominus \mathcal{X}_{1}\left(\varepsilon_{1 b}\right)\right)$ and $\left\|x(t)-\zeta_{o b s}(t)\right\| \leq \varepsilon_{o b s}$ for all $t \geq t_{o b s}=0.0255$. From (33), it follows that the above requirements are satisfied by setting $c=24.9, \theta=600$, and $\varepsilon_{o b s}=0.4562$.

Assumptions 1, 2, 3, and 4 hold, and in addition, it is satisfied $\mathcal{P}_{0}\left(\varepsilon_{0} \alpha\right) \subset \mathcal{X}_{1}\left(\varepsilon_{I}\right)$ with $\alpha=2.42$. Therefore, Theorem 1 guarantees that the hybrid output feedback controller $(\mathcal{C}, \mathcal{D}, u, v, w)$ solves the uniting problem with $\mathcal{B}=\Omega_{0}(1)$.

It should be pointed out that superior performance of the hybrid control system over (3) is not guaranteed. To show the benefits of the hybrid controller, we consider several levels of the Lyapunov function $V_{1}$ and a set of initial conditions uniformly distributed in each level (1000 initial conditions, $x_{0}^{j}, j \in\{1,2, \ldots, 1000\}$, for each level) with $q^{0}=0, \zeta_{0}^{0}=0$, and $\zeta_{o b s}^{0}=0$. Let $J_{R}\left(x_{0}\right)$ and $J_{U}\left(x_{0}\right)$ be the value of the cost function (25) evaluated along the solution to system (3) and (4), respectively, with initial condition $\left(x_{0}, 0\right)$. To highlight the improvement of the hybrid controller over the regional controller, the following improvement factor (IF) is considered.

$$
I F=\frac{J_{R}\left(x_{0}^{1}\right)+J_{R}\left(x_{0}^{2}\right)+\cdots+J_{R}\left(x_{0}^{1000}\right)}{J_{U}\left(x_{0}^{1}\right)+J_{U}\left(x_{0}^{2}\right)+\cdots+J_{U}\left(x_{0}^{1000}\right)} .
$$

The hybrid controller overcomes the performance of the system (3) for approximately the $99 \%$ of the initial conditions. Figure 2 shows the improvement factor as a function of the size of the set of initial conditions. As it may be expected, the improvement factor increases as the initial conditions approaches the origin. Finally, consider the initial condition $\left(x^{0}, \zeta_{0}^{0}\right)=$ $[0.682,1.191,-0.212,0,0,0]^{\top}$, then the simulation of the system (2) shows that the trajectory diverges. Hence, this initial condition does not belong to the domain of attraction. However, it is satisfied $\left[x^{0}, 0,0,0\right]^{\top} \in$ $\Omega_{1}\left(\varepsilon_{I}\right)$, and thus, the uniting controller stabilizes the system (22) with initial conditions for which the local controller cannot stabilize it. In order to observe the robustness of the uniting controller and quantify the deterioration of the performance in presence of uncertainty, we consider the following perturbed system: $f(x, u)=f_{n}(x)+(A+E) x+B \operatorname{sat}(u)$, where $E$ is an additive uncertainty (randomly computed) given by

$$
E=\left[\begin{array}{ccc}
-0.02 & -0.01 & -0.02 \\
0.02 & -0.04 & -0.01 \\
0.02 & 0.01 & 0.01
\end{array}\right]
$$

Figure 2 shows the improvement factor (36) for the perturbed system. Note that despite the uncertainty, the 


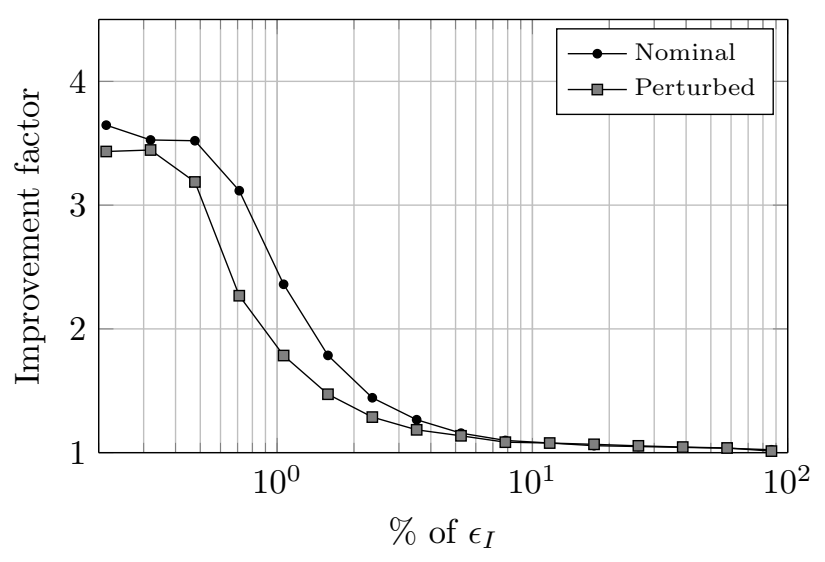

Fig. 2. Improvement factor as a function of the size of the set of initial conditions.

uniting controller improves the performance with respect to the regional controller for almost all the initial conditions $(96 \%$ of the initial conditions in the worst case).

\section{Conclusion}

For a given nonlinear system, two local feedback controllers with different objectives are assumed known: one renders the system local asymptotic stable with some desired performance, and the other provides local attractivity with a larger domain of attraction. In this context, we have posed the problem of enlarging a given estimation of the domain of attraction of the control system while the desired performance is guaranteed locally. The proposed solution consists of a hybrid output feedback controller formed as combination of both predesigned controllers. The event dynamics of the hybrid system is governed by a tunable observer. A motivating example, for which no previous solutions are applicable due to the input saturation, shows the effectiveness of the proposed solution.

\section{References}

[1] V. Andrieu, G. Besançon, and U. Serres. Observability necessary conditions for the existence of observers. In 52nd IEEE Conference on Decision and Control, pages 4442-4447, Florenze, Italy, 2013.

[2] V. Andrieu and C. Prieur. Uniting two control lyapunov functions for affine systems. IEEE Transactions on Automatic Control, 55(8):1923-1927, 2010.

[3] G. Besançon. Nonlinear observers and applications, volume 363. Springer Verlag, 2007.

[4] D. V. Efimov. Uniting global and local controllers under acting disturbances. Automatica, 42(3):489-495, 2006.

[5] D. V. Efimov, A. Loria, and E. Panteley. Robust output stabilization: improving performance via supervisory control. International Journal of Robust and Nonlinear Control, 21(10):1219-1236, 2011.
[6] J. P. Gauthier, H. Hammouri, and S. Othman. A simple observer for nonlinear systems applications to bioreactors. IEEE Transactions on Automatic Control, 37(6):875-880, 1992.

[7] R. Goebel, R. G. Sanfelice, and A. R. Teel. Hybrid dynamical systems: modeling, stability, and robustness. Princeton University Press, 2012.

[8] H.K. Khalil. Nonlinear Systems. Prentice-Hall, 3rd edition, 2002.

[9] S. Marx, V. Andrieu, and C. Prieur. Semi-global stabilization by an output feedback law from a hybrid state controller. Automatica, 74:90-98, 2016.

[10] F. Mazenc, L. Praly, and W. P. Dayawansa. Global stabilization by output feedback: examples and counterexamples. Systems \& Control Letters, 23(119-125), 1994

[11] P. Morin, R. M. Murray, and L. Praly. Nonlinear rescaling of control laws with application to stabilization in the presence of magnitude saturation. In 4th IFAC symposium on nonlinear control systems, Enschede, The Netherlands, 1998.

[12] Z. Pan, K. Ezal, A. J. Krener, and P. V. Kokotovic. Backstepping design with local optimality matching. IEEE Transactions on Automatic Control, 46(7):1014-1027, 2001.

[13] C. Prieur. Uniting local and global controllers with robustness to vanishing noise. Mathematics of Control, Signals, and Systems, 14:143-172, 2001.

[14] C. Prieur and A. R. Teel. Uniting local and global output feedback controllers. IEEE Transactions on Automatic Control, 56(7):1636-1649, 2011.

[15] R. G. Sanfelice and C. Prieur. Robust supervisory control for uniting two output-feedback hybrid controllers with different objectives. Automatica, 49(7):1958-1969, 2013.

[16] E. D. Sontag and H. J. Sussmann. Nonlinear output feedback design for linear systems with saturating controls. In 29th Conference on Decision and Control, pages 3414-3416, 1990.

[17] A. R. Teel, O. E. Kaiser, and R. M. Murray. Uniting local and global controllers for the caltech ducted fan. In American Control Conference, pages 1539-1543, Albuquerque NM, 1997.

[18] A. R. Teel and N. Kapoor. Uniting local and global controllers. In European Control Conference, Bruxelles, Belgium, 1997.

[19] A. R. Teel and L. Praly. Global stabilizability and observability imply semi-global stabilizability by output feedback. Systems \&3 Control Letters, 22:313-325, 1994. 


\section{University Library}

\section{- M M N E R VA A gateway to Melbourne's research publications}

Minerva Access is the Institutional Repository of The University of Melbourne

Author/s:

Davo, MA;Prieur, C;Fiacchini, M;Nesic, D

Title:

Enlarging the basin of attraction by a uniting output feedback controller

Date:

2018-04-01

Citation:

Davo, M. A., Prieur, C., Fiacchini, M. \& Nesic, D. (2018). Enlarging the basin of attraction by a uniting output feedback controller. Automatica, 90, pp.73-80. https://doi.org/10.1016/ j.automatica.2017.12.044.

Persistent Link:

http://hdl.handle.net/11343/251859 